\title{
Performance Analysis of Orthogonal Space-Time Block Code over Rayleigh Fading Channels for Various Modulation Techniques
}

\author{
Krishna Kumar Gupta \\ University College of Engineering, \\ Jawaharlal Nehru Technical University, \\ Kakinada, India
}

\author{
Leela Kumari Balivada \\ University College of Engineering, \\ Jawaharlal Nehru Technical University, \\ Kakinada, India
}

\begin{abstract}
The use of multiple antennas at both transmitter and receiver has been shown to be an effective way to improve capacity and reliability over those achievable with single antenna wireless systems. Orthogonal Space-Time Block Code (OSTBC) is space-time coding techniques in which data is split into $\mathrm{n}$ streams which are simultaneously transmitted using $\mathrm{n}$ transmit antennas which can be received by single or multiple antennas. The performance of OSTBC technique can be analyzed for different modulation techniques using multiple transmitting and receiving antennas in terms of BER \& SNR.
\end{abstract}

\section{General Terms}

Alamouti's algorithm, Encoding matrix.

\section{Keywords}

Rayleigh fading channel, Multiple Input Multiple Output (MIMO), Maximum Likelyhood decoding, Space-Time Block Coding

\section{INTRODUCTION}

Space-time block coding (STBC) $[1,2]$ technique has been implemented in current scenario of wireless communication .it combines coding, modulation, and uses antennas at both transmit and receive terminals. Using the demerits of wireless transmission of signals which is fading can be overcome by using MIMO [3-5] technique. The use MIMO is for increasing capacity. Higher order modulation technique increases the effective data rate.

Alamouti's et. Al [6], proposed a scheme, which uses less number of antenna at transmitter and receiver and gives the diversity [7, 8] advantage to the MIMO system with less complexity. This needs encoding [9] of signals at transmitters which can be decoded using some decoding technique at the receiver side.

By increasing the number of transmitter and receiver antenna yield more performance enhancement and diversity advantage with increased decoding complexity. So Orthogonal SpaceTime Block Coding (OSTBC) technique can be used in which principle orthogonality is to be followed between the signals from each transmitting antenna. This makes detection of signal linear and the signal can be detected singly which decreases the decoding complexity.

By using OSTBC [10-13] transmission rate can be increased and also the complexity of the decoding is decreased.

The channel type used is of Rayleigh fading channel [14, 15]. Topic to be discussed are alamouti's STBC as well as
Orthogonal STBC for 4 transmit antennas and do simulation and performance analysis.

The paper is organized as follows. In section 2, system model about MIMO. Next different STBC technique with encoding and decoding algorithm is to be explained in section 3.The simulation and Performance analysis in section 4 and finally section 5 will conclude.

\section{SYSTEM MODEL}

The system of multiple antennas consists of three main elements that are transmitter $(\mathrm{Tx})$, channel $(\mathrm{H})$ and receiver

$(\mathrm{Rx})$. The block diagram of system model is shown below,

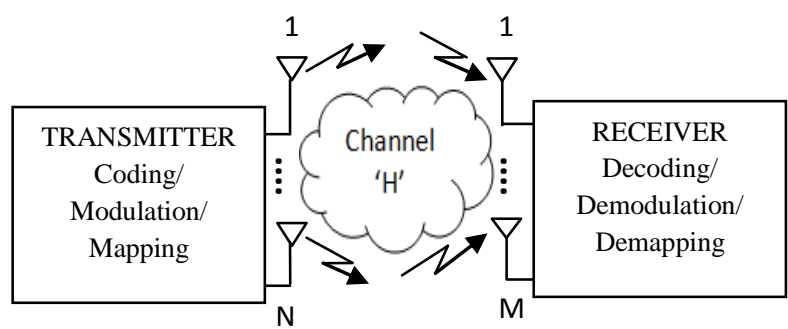

Figure 1: System block diagram

At the transmitter there are $1,2,3 \ldots \ldots . . \mathrm{N}$ transmitting antennas and at the receiver there are $1,2,3 \ldots . . . \mathrm{M}$ receiving antennas. Before transmission of signal from $\mathrm{N}$ transmitter they are encoded using principle of orthogonality ,after that are modulated using any of BPSK, QPSK, QAM or any other else modulation technique due to the reason of high transmission rate .These signals are then mapped on the basis of their signal constellations based on employed modulation scheme.

At the receiver side the same process reverses and the signal is received by $\mathrm{M}$ receiver are demapped, demodulated and decoded and finally required signal is obtained. The channel ' $\mathrm{H}$ ' is wireless medium through which signal transmit from transmitters and reach to receivers. The channel matrix can be represented as,

$$
\mathrm{H}=\left(\begin{array}{cccc}
\alpha_{1,1} & \alpha_{2,1} & \ldots & \alpha_{i, 1} \\
\alpha_{1,2} & \alpha_{2,2} & \ldots & \alpha_{i, 2} \\
\vdots & \vdots & \ddots & \vdots \\
\alpha_{1, j} & \alpha_{2, j} & \ldots & \alpha_{i, j}
\end{array}\right)
$$

Where $\alpha_{i, j}$ represents path gain from transmit antenna $i$ to receive antenna $j$. The path gains are considered to be independent complex Gausian random variables with variance 0.5 . The channel $(\mathrm{H})$ is to be considered as a quasi-static Rayleigh fading .it means path gain will be constant within a 
frame and vary from frame to frame of length ' $l$ '. The overall MIMO signal model is described as ,

$$
r_{i}^{j}=\sum_{i=1}^{n} \alpha_{i, j} c_{j}^{i}+n_{t}^{j}
$$

Where,

$r_{i}^{j}=$ received signal at receiver antenna $j$ at time slot $t$.

$\alpha_{i, j}=$ path gain from transmit antenna $i$ to receive antenna $j$.

$c_{t}^{i}=$ signal transmitted from transmit antenna $i$ at time slot $t$

$n_{t}^{j}=$ noise at receive antenna $j$ at time slot $t$.

Every noise is modeled as independent identically distributed AWGN random variable with variance $\mathrm{N} /(2 \mathrm{SNR})$. The average energy of the symbols transmitted from each transmitting antenna is one. The maximum likelihood detector at receiver computes the decision metric,

$$
\sum_{t=1}^{l} \sum_{j=1}^{m}\left|r_{i}^{j}-\sum_{i=1}^{n} \alpha_{i, j} c_{j}^{i}\right|^{2}
$$

Over all code words is,

$$
c_{1}^{1} c_{1}^{2} \ldots \ldots . c_{1}^{n} c_{2}^{1} c_{2}^{2} \ldots \ldots c_{2}^{n} \ldots . c_{l}^{1} c_{l}^{2} \ldots . c_{l}^{n}
$$

The ML detector makes a decision in favor of code word that minimizes above sum (2).

\section{SPACE-TIME BLOCK CODING}

\subsection{Encoding}

In a space time block coding scheme the signal are encoded in a transmission matrix $G$. The matrix will be of order $t \times \mathrm{N}$ where $t$ is $1,2,3 \ldots \ldots . t$ time slot and $\mathrm{N}$ is the number of transmitting antennas. The matrix constitutes linear combinations of signals variables $\mathrm{x}_{1}, \mathrm{x} 2, \mathrm{x} 3 \ldots \ldots . . \mathrm{x}_{\mathrm{k}}$ and their conjugates. Considering for two transmitting antennas, the transmission matrix for $G_{2}[16]$ is represented as,

$$
G_{2}=\left(\begin{array}{rr}
x_{1} & x_{2} \\
-x_{2}^{*} & x_{1}^{*}
\end{array}\right)
$$

Where, rows represents time slot ' $t$ ' and columns represents transmitting antennas. Signal transmission employs signals constellation with $2^{\mathrm{b}}$ elements, where $\mathrm{b}$ is number of bits for employed modulation scheme.

In Alamouti's et al, uses two transmit antennas regardless of the number of receive antennas. The transmission matrix is $G_{2}$ represented as,

$$
G_{2}=\left(\begin{array}{rr}
s_{1} & s_{2} \\
-s_{2}^{*} & s_{1}^{*}
\end{array}\right)
$$

Where, $s_{1}$ and $s_{2}$ are signals and $s_{1}^{*}$ and $s_{2}^{*}$ are respective conjugate of $s_{1}$ and $s_{2}$.

In this the row represents transmission of signal at different time slot and columns represents transmission of signal through different antennas .These orthogonality of signal allows a simple decoding scheme. In matrix $G_{2}, t$ time slots are used to transmit $k$ symbols.

Symbol Rate of any block code is given by the formula:
Symbol Rate $(R)=\frac{\text { No.of symbol transmitted }(k)}{\text { No.of time slots }(t)}$

where, $k$-number of transmitting symbols and

$t$ - number of time slots

For $G_{2}$ the rate $R=2 / 2$, so rate of $G_{2}$ is 1 .

For four transmitting antennas $G_{4}[16]$ is a transmission matrix and is represented as,

$$
G_{4}=\left(\begin{array}{rrrr}
s_{1} & s_{2} & s_{3} & s_{4} \\
-s_{2} & s_{1} & -s_{4} & s_{3} \\
-s_{3} & s_{4} & s_{1} & -s_{2} \\
-S_{4} & -s_{3} & s_{2} & s_{1} \\
s_{1}^{*} & s_{2}^{*} & s_{3}^{*} & s_{4}^{*} \\
-s_{2}^{*} & s_{1}^{*} & -s_{4}^{*} & s_{3}^{*} \\
-s_{3}^{*} & s_{4}^{*} & s_{1}^{*} & -s_{2}^{*} \\
-s_{4}^{*} & -s_{3}^{*} & s_{2}^{*} & s_{1}^{*}
\end{array}\right)
$$

The code rate of $G_{4}, R=1 / 2$.

\subsection{Decoding}

Decoding is performed at the receiver side. For linear processing of signals maximum likelihood decoding is employed in space time block code. In case of space time block code $G_{2}$, if there are $2^{\mathrm{b}}$ signals in the constellation ,then at time slot one $2 \mathrm{~b}$ bits arrive at the encoder and select two complex symbols $s_{1}$ and $s_{2}$. The symbols $s_{1}$ and $s_{2}$ are simultaneously transmitted from antenna one and two respectively.

The maximum likelihood detector minimizes the decision metric [16] over all possible values of signals $s_{1}$ and $s_{2}$.

$\sum_{j=1}^{m}\left(\left|r_{1}^{j}-\alpha_{1, j} s_{1}-\alpha_{2, j} s_{2}\right|^{2}+\left|r_{2}^{j}-\alpha_{1, j} s_{2}^{*}-\alpha_{2, j} s_{1}^{*}\right|^{2}\right)$

The minimized value of $s_{1}$ and $s_{2}$ at receiver is the estimates of $s_{1}$ and $s_{2}$. After expanding and deleting the independent terms of the codewords of the above eq. (6) the minimization will be equivalent to [16],

$$
\begin{aligned}
& \sum_{j=1}^{m}\left[r_{1}^{j} \alpha_{1, j}^{*} s_{1}^{*}+\left(r_{1}^{j}\right)^{*} \alpha_{1, j} s_{1}+r_{1}^{j} \alpha_{2, j}^{*} s_{2}^{*}+\left(r_{1}^{j}\right)^{*} \alpha_{2, j} s_{2}-\right. \\
& \left.r_{2}^{j} \alpha_{1, j}^{*} s_{2}-\left(r_{2}^{j}\right)^{*} \alpha_{1, j} s_{2}^{*}+r_{2}^{j} \alpha_{2, j}^{*} s_{1}+\left(r_{2}^{j}\right)^{*} \alpha_{1, j} s_{1}^{*}\right]+ \\
& \left(\left|s_{1}\right|^{2}+\left|s_{2}\right|^{2}\right) \sum_{j=1}^{m} \sum_{i=1}^{2}\left|\alpha_{i, j}\right|^{2}
\end{aligned}
$$

The above equation decomposes in two parts one of which is a function of $s_{1}$ as [16],

$$
\begin{aligned}
& -\sum_{j=1}^{m}\left[r_{1}^{j} \alpha_{1, j}^{*} s_{1}^{*}+\left(r_{1}^{j}\right)^{*} \alpha_{1, j} s_{1}+r_{2}^{j} \alpha_{2, j}^{*} s_{1}+\left(r_{2}^{j}\right)^{*} \alpha_{2, j} s_{1}^{*}\right]+ \\
& \left|s_{1}\right|^{2} \sum_{j=1}^{m} \sum_{i=1}^{2}\left|\alpha_{i, j}\right|^{2}
\end{aligned}
$$

And as below other is function of $s_{2}[16]$. 
$-\sum_{j=1}^{m}\left[r_{2}^{j} \alpha_{2, j}^{*} s_{2}^{*}+\left(r_{1}^{j}\right)^{*} \alpha_{2, j} s_{2}-r_{2}^{j} \alpha_{1, j}^{*} s_{2}-\left(r_{2}^{j}\right)^{*} \alpha_{1, j} s_{2}^{*}\right]+$ $\left|s_{2}\right|^{2} \sum_{j=1}^{m} \sum_{i=1}^{2}\left|\alpha_{i, j}\right|^{2}$

Then for detecting $s_{1}$ above equation is equivalent to minimizing the decision metric to,

$\left|\left[\sum_{j=1}^{m}\left(r_{1}^{j} \alpha_{1, j}^{*}+\left(r_{2}^{j}\right)^{*} \alpha_{2, j}\right)\right]-s_{1}\right|^{2}+(-1+$

$\left.\sum_{j=1}^{m} \sum_{i=1}^{2}\left|\alpha_{i, j}\right|^{2}\right)\left|s_{1}\right|^{2}$

and for detecting $s_{2}$ above equation is equivalent to minimizing the decision metric to,

$\left|\left[\sum_{j=1}^{m}\left(r_{1}^{j} \alpha_{2, j}^{*}-\left(r_{2}^{j}\right)^{*} \alpha_{1, j}\right)\right]-s_{2}\right|^{2}+(-1+$

$\left.\sum_{j=1}^{m} \sum_{i=1}^{2}\left|\alpha_{i, j}\right|^{2}\right)\left|s_{2}\right|^{2}$

Similarly, the decoders for $G_{4}$ can be obtained.

\section{SIMULATION RESULTS ANALYSIS}

AND

Simulations are done in MATLAB [17] using Rayleigh fading channel. From the below figure 2 and 3 using $G_{2}$ matrix for 1 and 2 receive antennas, it is observed that as number of receiving antenna increases at particular BER the SNR value decreases, that is power of signal transmission requirement is less.

In both figure 2 and 3 BPSK has gain over QPSK, 16-QAM and 64-QAM. At BER $10^{-4}$ BPSK have $5 \mathrm{~dB}, 16 \mathrm{~dB}$ and $28 \mathrm{~dB}$ gain over QPSK, 16QAM and 64QAM.

In figure 4,5,6 and 7 simulation are done using four transmitting antennas and 1,2,3 and 4 receiving antennas respectively using $G_{4}$ as encoding matrix. It is observed that as number of receiving antennas increases at particular BER the SNR value decreases. In all figure 4, 5, 6 and 7 BPSK has gain over QPSK, 16QAM and 64QAM.

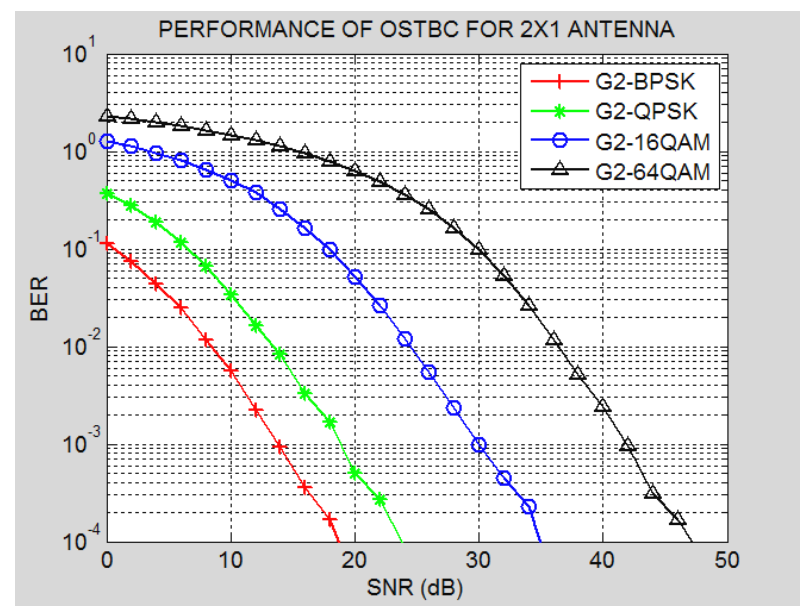

Figure 2: Two transmit and one receive antenna

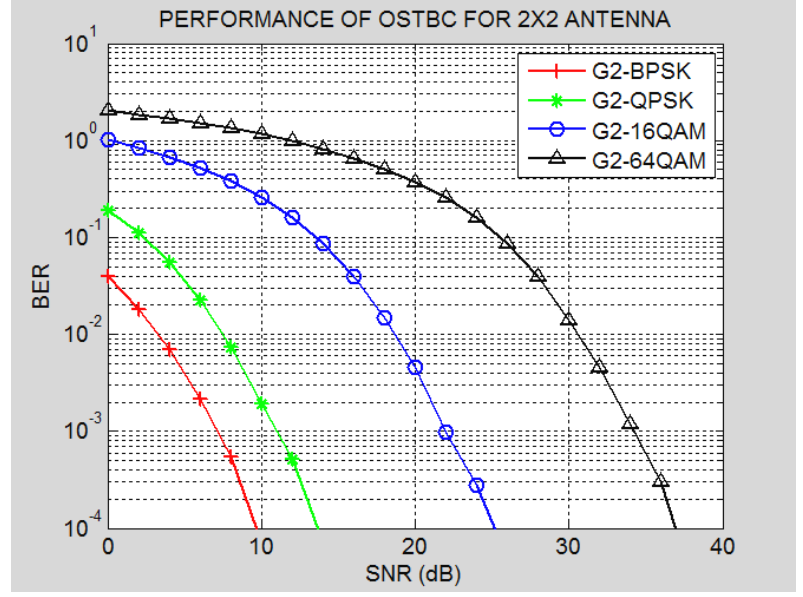

Figure 3: Two transmit and two receive antenna

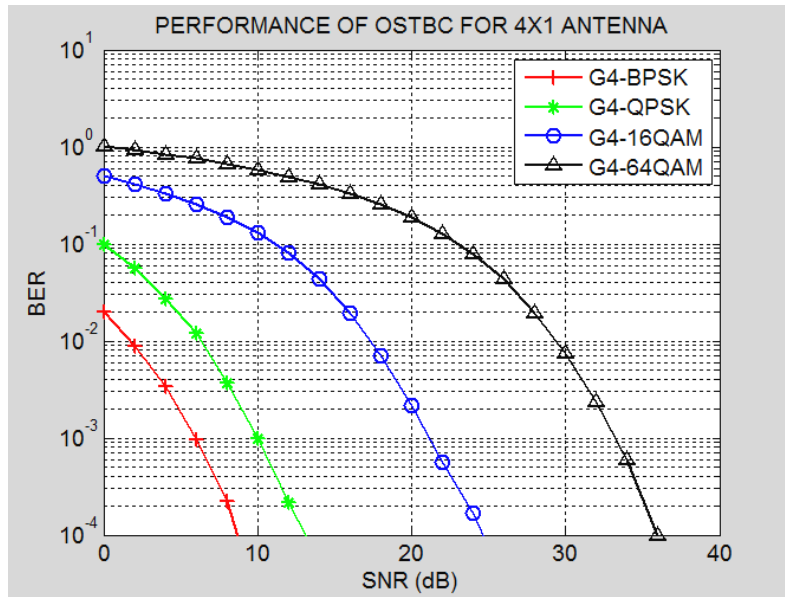

Figure 4: Four transmit and one receive antenna

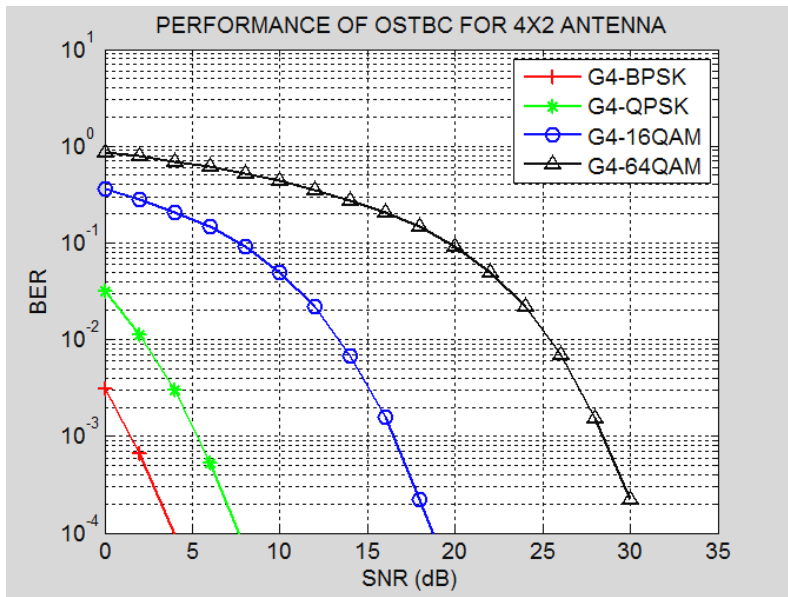

Figure 5: Four transmit and two receive antenna 


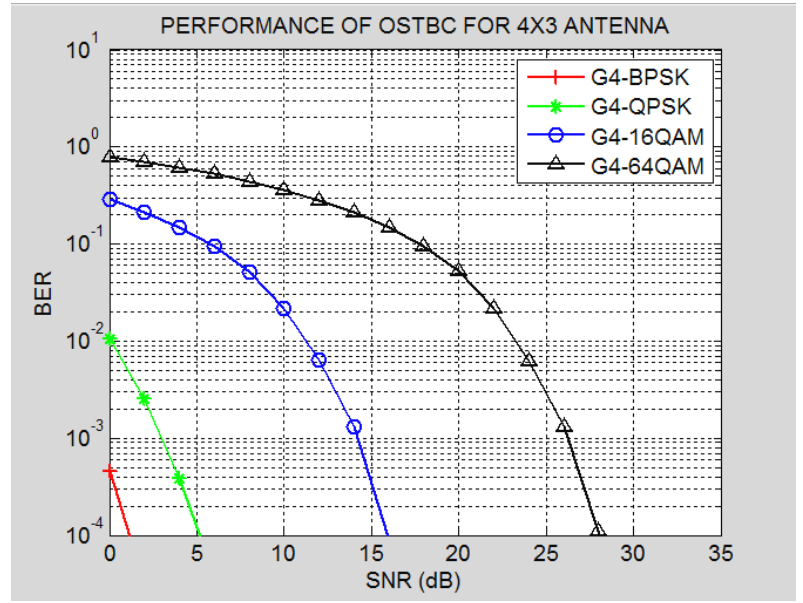

Figure 6: Four transmit and three receive antenna

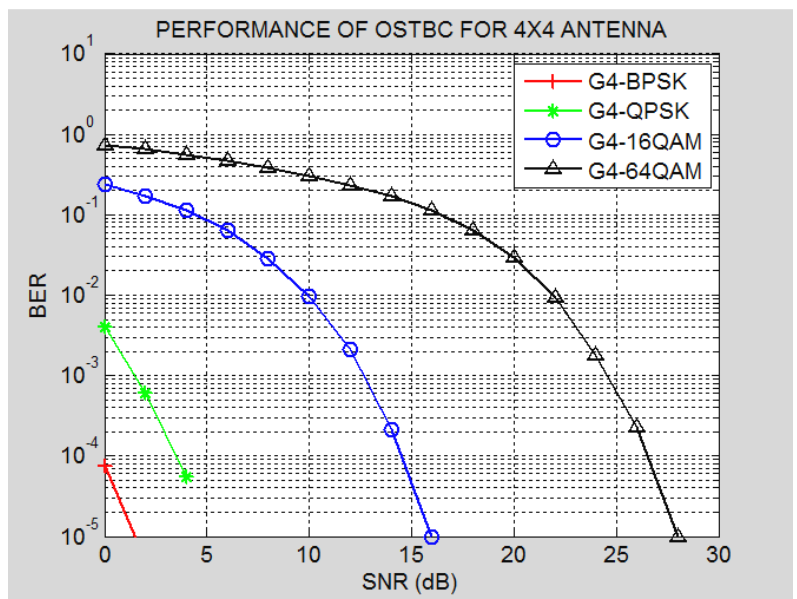

Figure 7: Four transmit and four receive antenna

The below figure 8 shows bar chart of SNR requirement for each modulation technique for various diversity order of the system at BER $10^{-4}$.

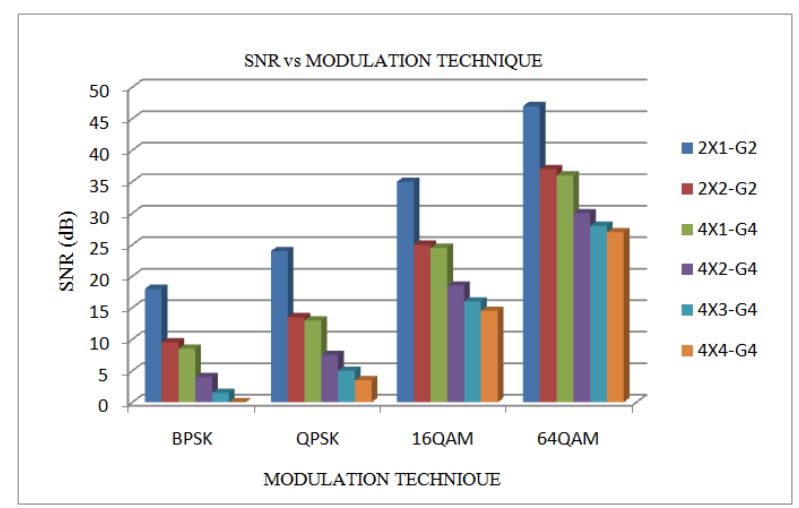

Figure 8: Performance of OSTBC at BER $10^{-4}$

The below figure 9 shows bar chart of SNR requirement for each modulation technique for various diversity order of the system at BER $10^{-3}$.

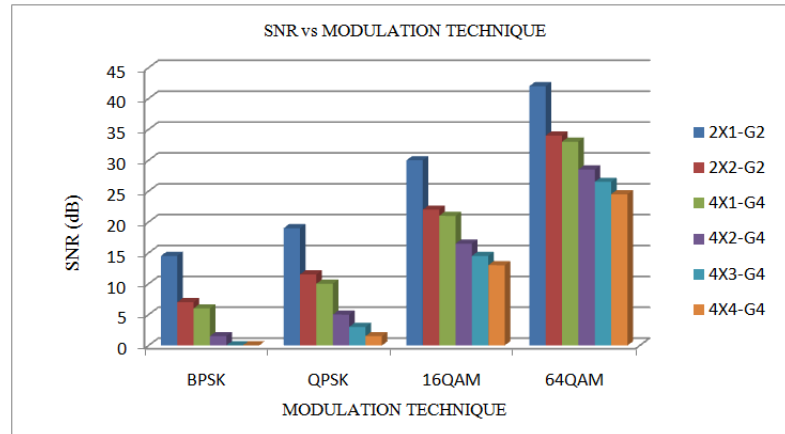

Figure 9: Performance of OSTBC at BER 10 ${ }^{-3}$

The below table 1 shows the total transmission rate for each system of antennas on the basis of code rate of $G_{2}$ and $G_{4}$ for each modulation technique.

Table 1. Total transmission rate for various antennas at transmitter and receiver

\begin{tabular}{|c|c|c|c|c|c|}
\hline \multirow{3}{*}{$\begin{array}{l}\text { Antennas } \\
(\mathrm{Nt} \times \mathrm{Nr})\end{array}$} & \multirow{3}{*}{$\begin{array}{l}\text { Code } \\
\text { rate }\end{array}$} & \multirow{2}{*}{\multicolumn{4}{|c|}{ Total transmission rate $(\mathrm{bits} / \mathrm{sec} / \mathrm{Hz})$}} \\
\hline & & & & & \\
\hline & & BPSK & QPSK & $\begin{array}{l}16- \\
\text { QAM }\end{array}$ & $\begin{array}{l}64- \\
\text { QAM }\end{array}$ \\
\hline $2 \mathrm{X} 1$ & 1 & 1 & 2 & 4 & 6 \\
\hline $2 \mathrm{X} 2$ & 1 & 1 & 2 & 4 & 6 \\
\hline $4 \mathrm{X} 1$ & $1 / 2$ & 0.5 & 1 & 2 & 3 \\
\hline $4 \mathrm{X} 2$ & $1 / 2$ & 0.5 & 1 & 2 & 3 \\
\hline $4 \mathrm{X} 3$ & $1 / 2$ & 0.5 & 1 & 2 & 3 \\
\hline $4 \mathrm{X} 4$ & $1 / 2$ & 0.5 & 1 & 2 & 3 \\
\hline
\end{tabular}

\section{CONCLUSION}

This paper provides the basic overview of the MIMO systems. The space-time block coding is provided for 2 and 4 transmitting antennas. Various modulation technique are employed to each configuration of number of transmit and receive antennas. It is observed that as diversity increases the performance of OSTBC (Orthogonal Space-Time Block Code) increases and requirement of power of signal for transmission is less. Also higher transmission rate can be achieved by employing higher order modulation technique.

\section{REFERENCES}

[1] Hamid Jafarkhani "Space-time coding theory and practice" Cambridge University Press 2005.

[2] David Gesbert, Mansoor Shafi,. From theory to practice: an overview of MIMO space-time coded wireless systems[J]. IEEE J. Select. Area.Commun., 2003, 21(3): 281-302R. Nicole, "Title of paper with only first word capitalized," J. Name Stand. Abbrev., in press.

[3] G. Foschini and M. Gans, "On limits of wireless communications in a fading environment when using multiple antennas," Wireless personal communications, vol. 6, no. 3, pp. 311-335, 1998.

[4] Michael Joham, Linear Transmit Processing in MIMO Communications Systems [J]. IEEE Transactions on SignalProcessing, 2005, 53(8): 2700-2712.

[5] A. Molisch, Wireless Communications. Wiley-IEEE Press, 2005. 
[6] S.M.Alamouti. A simple transmit diversity scheme for wireless communication[J]. IEEE J. Select.Area. Commun. , Oct 1998, 16(8): 1451-1458.

[7] J. Winters, J. Salz, and R. D. Gitlin, "The impact of antenna diversity on the capacity of wireless communication systems," IEEE Trans.Commun., vol. 42. no. 2/3/4, pp. 1740-1751, Feb./Mar./Apr. 1994.

[8] H. Niu and Ngo, "Diversity and Multiplexing Switching in 802.11 n MIMO Systems," in Signals, Systems and Computers, 2006. ACSSC'06. Fortieth Asilomar Conference on, 2006, pp. 1644-1648.

[9] V.Tarokh, H.Jafarkhani and A.R.Calderbank. Space-time block codes from orthogonal designs[J]. IEEE Trans. On IT., July 1999, 45(5): 1456-1467.

[10] Tarokh V, Seshadri N, Calderbank A R. Space-time codes for high data rate wireless communication: performance criterion and code construction[J]. IEEE Trans.on I.T., 1998, 44(2):744-765.

[11] A.F.Naguib, R.Calderbank. Space-time cods for high data rate wireless communications[J]. IEEE Signal Processing Magazine, 17(3): 76-92.

[12] V.Tarokh, H.Jafarkhani and A.R.Calderbank, The application of orthogonal designs to wireless communication," in Proc. IEEE Information Theory Workshop, Killarney, Ireland, June 1998, pp. 46-47.

[13] Zhang Zhen-chuan, Li Ying Research on OSTBC Over Rayleigh Fading Channels and Its Performance Simulation IEEE Conf, 978-1-4244-3709-2/10.

[14] J. Winters, "On the capacity of radio communication systems with diversity in a Rayleigh fading environment, "IEEE Journal on Selected Areas in Communications, vol. 5, no. 5, pp. 871-878, 1987.

[15] J.-C. Guey, M. P. Fitz, M. R. Bell, and W.-Y. Kuo, "Signal design for transmitter diversity wireless communication systems over Rayleigh fading channels," in Proc. IEEE VTC'96, Apr. 1996, pp. 136-140.

[16] Tarokh V,Jafarkhani H,Calderbank A R. Space-time block coding for wireless communications: performance results[J]. IEEE Journalon selected areas in communications, 1999, 17(3): 451-460.

[17] Rudra Pratap, "Getting started with MATLAB" 2009. Oxford University Press 2009 